\title{
Comprehensive analysis of a microRNA expression profile in pediatric medulloblastoma
}

\author{
JUNQIANG DAI $^{1 *}$, QIAO $^{1}{ }^{1 *}$, ZHITONG BING $^{2}$, YINIAN ZHANG ${ }^{1}$, LIANG NIU $^{1}$, \\ HANG YIN $^{1}$, GUOQIANG YUAN ${ }^{3}$ and YAWEN PAN ${ }^{1,3}$ \\ ${ }^{1}$ Department of Neurosurgery, Lanzhou University Second Hospital, Lanzhou, Gansu 730030; \\ ${ }^{2}$ Institute of Modern Physics, Chinese Academy of Sciences, Lanzhou, Gansu 730000; \\ ${ }^{3}$ Institute of Neurology, Lanzhou University Second Hospital, Lanzhou, Gansu 730030, P.R. China
}

Received March 10, 2016; Accepted February 23, 2017

DOI: $10.3892 / \mathrm{mmr} .2017 .6490$

\begin{abstract}
Medulloblastoma is the most common malignant brain tumor of the central nervous system among children. Medulloblastoma is an embryonal tumor, of which little is known about the pathogenesis. Several efforts have been made to understand the molecular aspects of its tumorigenic pathways; however, these are poorly understood. microRNA (miRNA), a type of non-coding short RNA, has been proven to be associated with a number of physiological processes and pathological processes of serious diseases, including brain tumors. Differentially expressed miRNAs serve an important role in numerous types of malignancy. The present study aims to define a differentially expressed set of miRNAs in medulloblastoma tumor tissue, compared with normal samples, to improve the understanding of the tumorigenesis. It was identified that 22 miRNAs were upregulated and 26 miRNAs were downregulated in the tumor tissue compared with the normal group. However, when the medulloblastoma tissue was compared with normal cerebellum tissue, 9 miRNAs were identified to be up or downregulated in the tumor samples. The differentially expressed miRNAs in the tumor tissue were identified in order to clarify the networks and pathways of tumorigenesis using Ingenuity Pathway Analysis. Subsequently, key regulatory genes associated with the development of medulloblastoma were identified, including tumor protein p53, insulin like growth factor 1 receptor, argonaute 2 , mitogen-activated protein kinases 1 and 3 , sirtuin 1 and $\mathrm{Y}$ box binding protein 1 .
\end{abstract}

Correspondence to: Professor Yawen Pan, Department of Neurosurgery, Lanzhou University Second Hospital, 82 Cuiyingmen Road, Chengguan, Lanzhou, Gansu 730030, P.R. China

E-mail: panyawen666@sohu.com

*Contributed equally

Key words: medulloblastoma, microRNA, microRNA profile, Gene Expression Omnibus database, Ingenuity Pathway Analysis

\section{Introduction}

Medulloblastoma is the most common pediatric brain tumor, with an incidence of $\sim 0.5 / 100,000$ children $<15$ years old (1). It is classified as grade IV according to the World Health Organization (WHO) (2). Current treatment consisting of surgery, radiotherapy and adjuvant chemotherapy has a limited role in improving the prognosis of patients $(3,4)$. Novel therapeutic methods based on the specific mechanism of medulloblastoma carcinogenesis are required to improve treatment efficiency and avoid the side effects of traditional therapy. An increasing number of studies have revealed that microRNAs (miRNAs/miRs) serve a role in tumorigenesis (5-9).

miRNAs are a type of small non-coding RNA (18-24 nucleotides). Gene expression is regulated by thousands of known miRNAs, which bind to imperfect complementary sites within the $3^{\prime}$ untranslated regions of their target protein-coding mRNAs, and repressing the expression of these genes at the level of mRNA stability and translation (10). Previous research has revealed that miRNAs are involved in the regulation of the majority of physiological and pathological process, including development, life span, and metabolism, and their dysregulation contributes to a number of types of disease, notably cancer (11). These small RNAs may function as oncogenes or tumor suppressors by modulating the levels of critical proteins, and their relevance in human disease and therapy is currently under investigation (12).

An increasing number of studies have demonstrated abnormal miRNA expression levels in the central nervous system (CNS) of cancer patients, suggesting that miRNAs may serve as key regulatory components in cancer of the CNS $(5,10,13)$. The present study analyzed the medulloblastoma miRNA expression profiles for pediatric brain tumors. It was observed that 22 miRNAs were upregulated and 26 miRNAs were downregulated in the tumor tissue. Ingenuity Pathway Analysis (IPA) was used to analyze the regulatory network of the differentially expressed miRNA in medulloblastoma. It was proposed that the pathways exhibited in the network may have an important role in the tumorigenesis of medulloblastoma. 


\section{Materials and methods}

The data were downloaded from the NCBI Gene Expression Omnibus (GEO) database (GSE42657, www.ncbi.nlm.nih. gov/geo). The series matrix file was downloaded and a $\log 2$ transformation was performed. The data include 7 control samples (consisting of 3 cerebellum and 4 frontal lobe controls) and 9 medulloblastoma samples. The data from all samples was normalized using the limma package in $\mathrm{R}$ software version 3.3.0 (https://www.r-project.org/). The selected samples of this profile are exhibited in Table I. The differentially expressed genes (DEGs) were obtained with the thresholds of $|\log F C|>1.0$ and $\mathrm{P}<0.01$, using linear models and empirical Bayes methods for assessing differential expression in microarray experiments in the limma package (Table II). A heat map of the different groups was produced to visualize the DEGs and cluster the corresponding groups with the differentially expressed miRNAs from the tumor tissue, using the $\mathrm{R}$ package gplots (Fig. 1).

The dataset (48 differentially expressed miRNAs) was uploaded to the Ingenuity Pathway Analysis software version 2016 (Qiagen, Inc., Valencia, CA, USA), using the microRNA target filter to find the targeting information. A total of 40 microRNAs targeting 11,757 mRNAs were identified by the filter. The core analysis function (rapid assessment of the signaling and metabolic pathways, molecular networks, and biological processes that are most markedly perturbed in the dataset of interest) to analyze the associated network functions of the miRNAs.

\section{Results}

Using miRNA microarray data from the GEO database, 48 miRNAs which are differentially expressed in medulloblastoma were identified, compared with the control group (Table II). The core analysis demonstrated the molecular network interactions and signaling pathways associated with 28 differentially expressed miRNAs of medulloblastoma, and their predicted molecular targets were rebuilt using IPA. The network 'Organismal Injury and Abnormalities, Reproductive System Disease, Cancer' had an IPA score of 41, focusing on 17 miRNA molecules, and the network 'Cancer, Organismal Injury and Abnormalities, Cell Death and Survival' had an IPA score of 23, focusing on 11 miRNA molecules (Fig. 2). The most impacted biological processes and diseases regulated by the analyzed miRNAs included organismal injury and abnormalities, reproductive system disease and cancer. The molecular network maps demonstrated that three primary components were identified to be at the central hub of the most significant network with a score of 41; these were tumor protein p53 (TP53), argonaute 2 (AGO2) and mitogen activated kinases 3 and 1 (ERK1/2) (Fig. 2A). TP53, sirtuin 1 (SIRT1) and Y box protein 1 (YBX1) were located in the center of the second network with a core analysis score of 23 (Fig. 2B). The first network included miR-17-5p, miR-130a-3p, miR-182-5p, miR-18a-5p, miR-19b-3p, miR-4288, miR-96-5p, which were upregulated in the medulloblastoma samples, and miR-128-3p, miR-132-3p, miR-133a-3p, miR-299a-5p, miR-409, miR-668-3p, which were downregulated. The second notable network (Fig. 2B) involved upregulated miR-217-5p
Table I. Sample descriptions.

\begin{tabular}{lcl}
\hline Diagnosis & $\begin{array}{c}\text { No. of } \\
\text { sample }\end{array}$ & \multicolumn{1}{c}{ Serial no. of sample } \\
\hline Normal (frontal lobe) & 4 & $\begin{array}{l}\text { GSM1047526,GSM1047529- } \\
\text { GSM1047531 } \\
\text { Normal (cerebellum) }\end{array}$ \\
GSM1047525,GSM1047527- & $\begin{array}{l}\text { GSM1047528 } \\
\text { GSM1047507-GSM1047515 }\end{array}$ \\
\hline
\end{tabular}

Table II. miRs significantly differentially expressed $(\mathrm{P}<0.01)$ in medulloblastoma tissue compared with normal control samples.

Gene names Log fold change

Upregulated miRs

hsa-miR-217 5.40

hsa-miR-301 2.54

hsa-miR-216 4.67

hsa-miR-19a

2.11

hsa-miR-15b

1.16

hsa-miR-106b

1.19

hsa-miR-18a

2.37

hsa-miR-130b

1.56

hsa-miR-182

3.52

hsa-miR-345

1.62

hsa-miR-17-3p

2.10

hsa-miR-96

3.71

hsa-miR-582

2.06

hsa-miR-622

2.57

hsa-miR-532

1.12

hsa-miR-19b

1.02

hsa-miR-18b

2.31

hsa-miR-632

2.52

hsa-miR-148a

1.13

hsa-miR-17-5p

2.99

hsa-miR-183

3.73

hsa-miR-135a

Downregulated miRs

hsa-miR-383

$-3.05$

hsa-miR-128a

$-2.53$

hsa-miR-433

$-2.38$

hsa-miR-488

$-2.22$

hsa-miR-584

$-2.97$

hsa-miR-128b

$-2.92$

hsa-miR-485-3p

$-1.89$

hsa-miR-329

$-2.43$

hsa-miR-299-5p

$-2.22$

hsa-miR-133b

$-1.60$

hsa-miR-330

$-2.37$

hsa-miR-181b

$-1.17$

hsa-miR-138

$-2.03$

hsa-miR-770-5p 
Table II. Continued.

\begin{tabular}{lc}
\hline Gene names & Log fold change \\
\hline hsa-miR-149 & -1.57 \\
hsa-miR-133a & -1.00 \\
hsa-miR-328 & -1.44 \\
hsa-miR-409-3p & -1.63 \\
hsa-miR-656 & -2.17 \\
hsa-miR-642 & -2.37 \\
hsa-miR-432 & -1.15 \\
hsa-miR-539 & -1.03 \\
hsa-miR-668 & -1.79 \\
hsa-miR-487b & -1.48 \\
hsa-miR-154* & -1.15 \\
hsa-miR-212 & -1.81 \\
\hline miR, microRNA. hsa-miR-154*, The precursor miRNA (pre-miRNA) \\
undergoes another endonucleolytic cleavage, which is catalysed by \\
Dicer, generating a miRNA-miRNA* duplex (where miRNA is the \\
antisense, or guide, strand and miRNA* is the sense, or passenger, \\
strand) of approx21-25 nucleotides (38). \\
\hline
\end{tabular}

Table III. miRs significantly differentially expressed $(\mathrm{P}<0.01)$ in medulloblastoma tissue compared with normal cerebellum samples.

\begin{tabular}{lc}
\hline Gene names & Log fold change \\
\hline $\begin{array}{l}\text { Upregulated miRs } \\
\text { hsa-miR-217 }\end{array}$ & 5.22 \\
hsa-miR-216 & 5.17 \\
hsa-miR-582 & 3.31 \\
hsa-miR-15b & 1.28 \\
Downregulated miRs & \\
hsa-miR-383 & -3.39 \\
hsa-miR-206 & -3.40 \\
hsa-miR-133b & -2.47 \\
hsa-miR-128a & -2.61 \\
hsa-miR-654 & -1.89 \\
\hline
\end{tabular}

miR, microRNA.

and miR-216a-5p, and downregulated miR-329, miR-330 and miR-584, which are associated with the regulation of key regulatory genes in medulloblastoma development, including TP53, SIRT1 and YBX1.

However, when the cerebellum samples were used as the control, it was observed that 4 miRNAs were overexpressed and 5 miRNAs were underexpressed in the medulloblastoma (Table III). The differentially expressed miRNAs were uploaded to the IPA and core analysis was performed. The network 'Cell death and Survival, Gastrointestinal Disease, Hepatic System Disease' had an IPA score of 9, focusing on 4 miRNA molecules (Fig. 3).
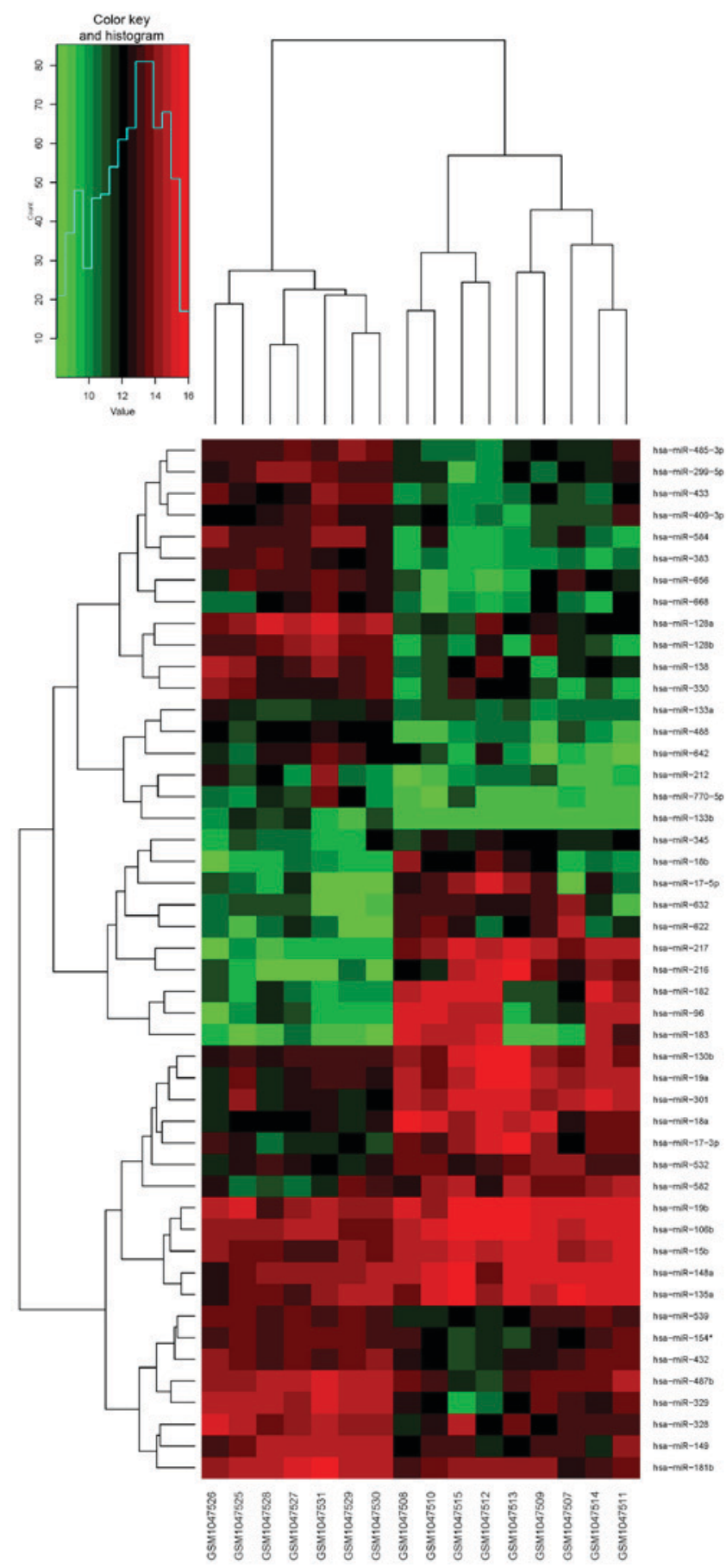

Figure 1. Differentially expressed miRs in medulloblastoma tissue compared with normal control tissue, visualized through clustering of sample data using the gplots package in $\mathrm{R}$ software. Red represents a miR expression level above the mean; green represents a miR expression level below the mean. The differentially expressed miRs were validated with the data in Gene Expression Omnibus database and the PubMed database articles. miRs miR-17-5p, miR-148a, miR-18a, miR-19a and miR-106a were co-upregulated in medulloblastoma. miR-383, miR-328, miR-128a/b, miR-133b, miR-149, miR-181b, miR-138 and miR-154 were co-downregulated in medulloblastoma. miR, microRNA.

\section{Discussion}

miRNAs function as master regulators and signal modulators by fine-tuning gene expression in multiple complex pathways. 


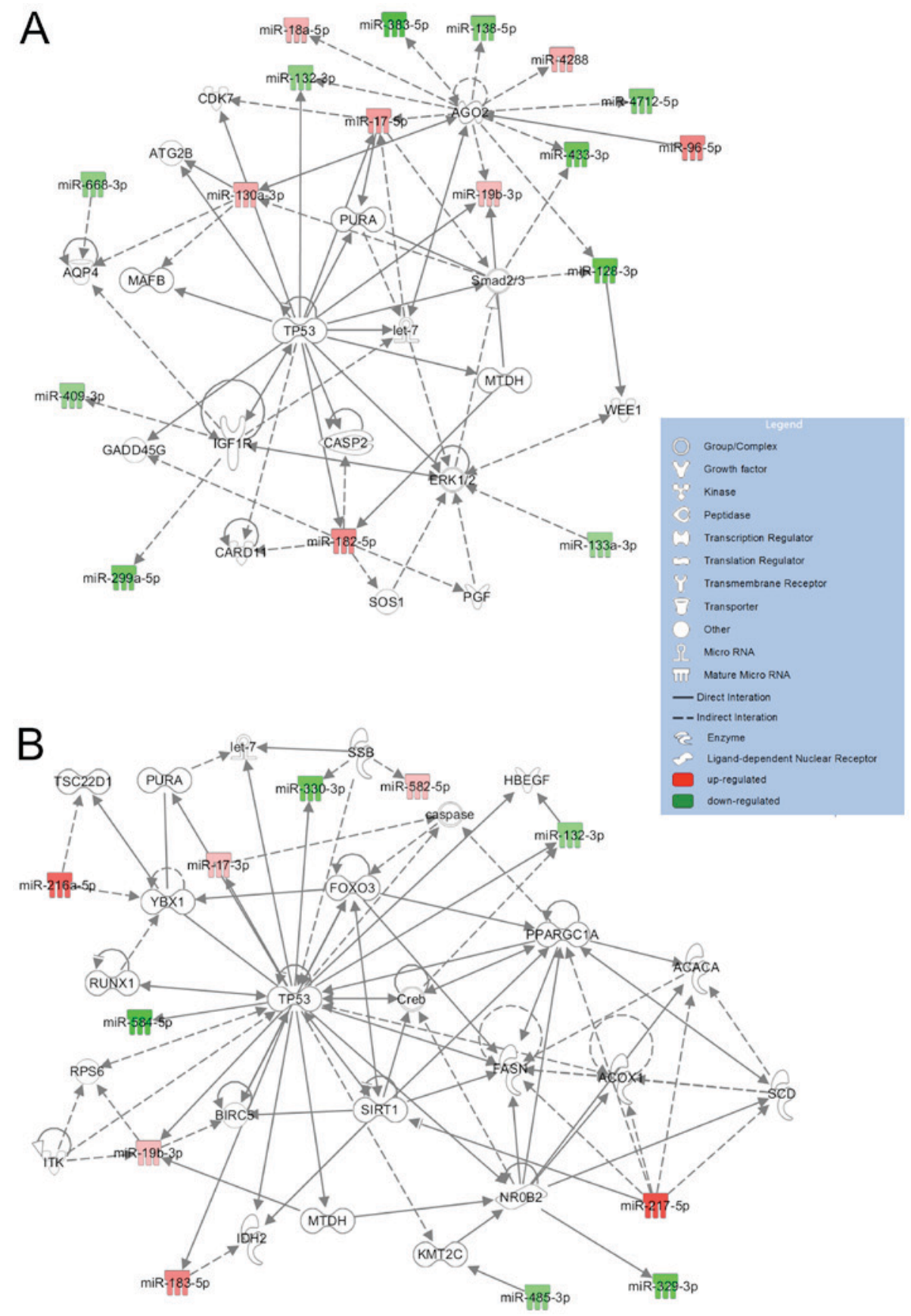

Figure 2. Representative network images of (A) 'Organismal Injury and Abnormalities, Reproductive System Disease, Cancer' with an IPA score of 41, focusing on 17 molecules, and (B) 'Cancer, Organismal Injury and Abnormalities, Cell Death and Survival' with an IPA score of 23, focusing on 11 molecules. IPA, Ingenuity Pathway Analysis.

Disruption of miRNAs may result in a permissive tumorigenic state (13). A number of the miRNAs which exhibit dysregulated expression in medulloepithelioma have been reported to serve various roles in carcinogenesis $(6,7,9)$. Through analysis of a GEO miRNA expression profiling dataset, miRNAs which are over- and underexpressed in medulloblastoma were identified, including miR-217, miR-216, miR-183, miR-182 and miR-96, which are upregulated in tumor tissue. Previous research demonstrates that miR-183-96-182 regulates cell survival, proliferation and migration in medulloblastoma, and increased miR-183-96-182 expression is associated with aggressive clinical course, high rates of metastasis and poor overall survival $(8,9,14)$.
miR-217 and miR-216 were overexpressed in medulloblastoma. However, the role of miR-217 was controversial. miR-217 is an oncogene that is overexpressed in aggressive human B cell lymphomas (15). However, it may be a tumor suppressor in hepatocellular carcinoma (16). In pancreatic intraepithelial neoplasm, pancreatic ductal adenocarcinomas and clear cell renal carcinomas, miR-217 was observed to be downregulated $(17,18)$. Further studies are required to determine whether increased expression of miR-217 in medulloblastoma represents an oncogenic effect, or if the dysregulation functions as a potential tumor suppressor. While miR-216 was underexpressed in various types of cancer, including breast cancer, pancreatic cancer and nasopharyngeal carcinoma (19-21), it may serve as 


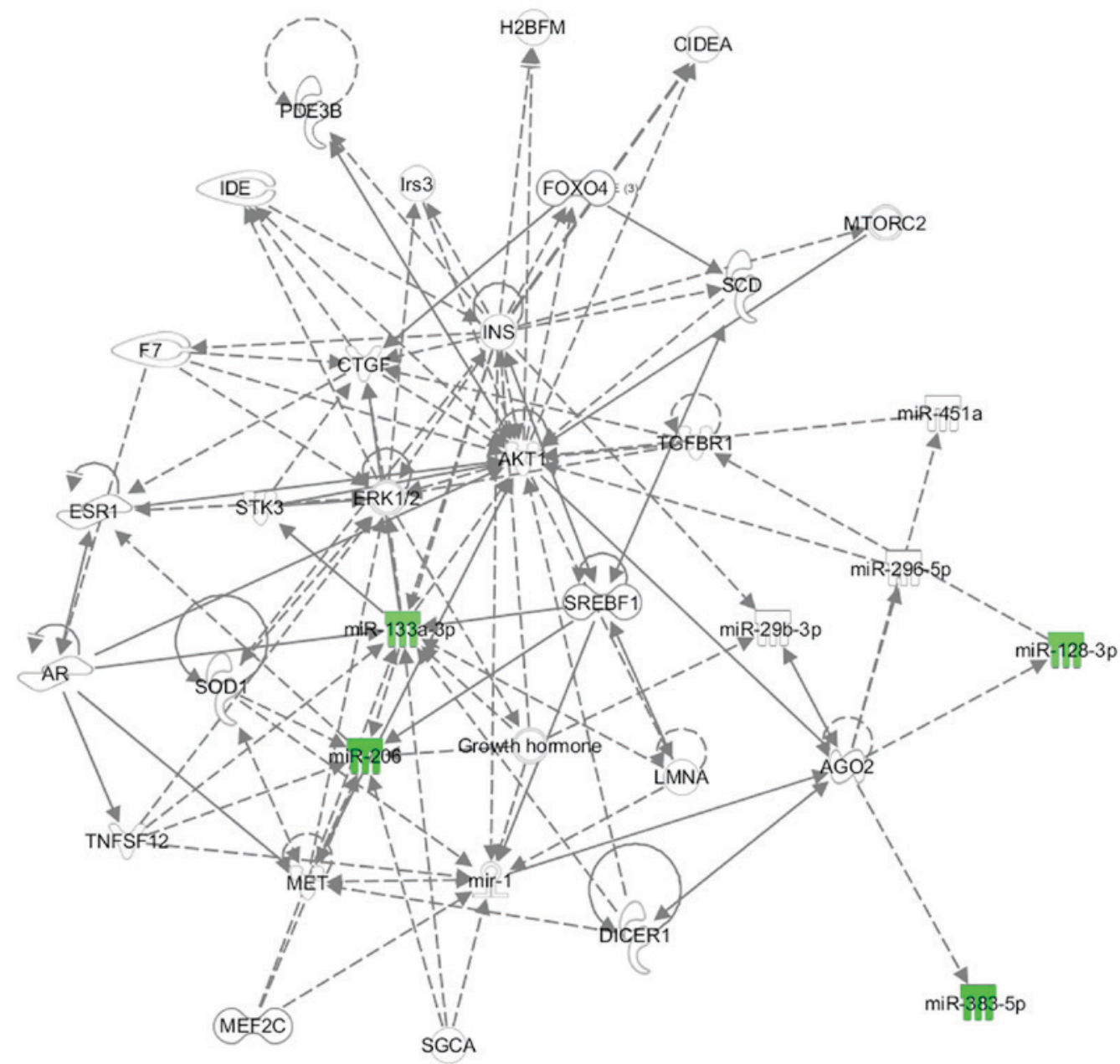

Figure 3. Differentially expressed miRs, comparing medulloblastoma with normal cerebellum tissue, revealing the network of 'Cell death and Survival, Gastrointestinal Disease, Hepatic System Disease' with an Ingenuity Pathway Analysis score of 9, focusing on four miR molecules. miR, microRNA.

a tumor suppressor to inhibit cell proliferation, invasion and tumor growth. Further studies are required to determine whether increased expression of miR-216 in medulloblastoma represents an oncogenic effect or is a potential tumor suppressor.

In the present study, marked downregulation of miR-383, miR-206, miR-138, miR-128a/b and miR-133b was identified. A previous study suggested that miR-383 is downregulated in medulloblastoma. miR-383, repressing peroxiredoxin 3 at transcriptional and translational levels, suppressed the cell growth of medulloblastoma (6). Additionally, miR-206 was downregulated in all the four molecular subgroups of medulloblastoma as well as in cell lines. Orthodenticle homeobox 2 (OTX2), a target gene of miR-206 which is overexpressed in all non-sonic-hedgehog-driven medulloblastomas, is an oncogene in medulloblastoma. Overexpressed OTX2 supported the growth and proliferation of medulloblastomas. Therefore, underexpression of miR-206 contributed to the upregulation of OTX 2 expression and enhanced growth of medulloblastoma cell lines (7). Studies have demonstrated a reduction in the miRNAs miR-383, miR-138, miR-128a/b, miR-133, miR-124 and let-7g in medulloblastoma. Nevertheless, miR-328, miR-133b, miR-149, miR-181b and miR-154 were downregulated in the study of Ferretti et al (5) and the present study. In addition, the present study revealed that miR-433, miR-488, miR-584, miR-329,
miR-299-5p, miR-330, miR-770-5p, miR-656 and miR-642 were underexpressed in medulloblastoma, and further research is required to identify whether these miRNAs have a specific role in the tumorigenesis of medulloblastoma.

Based on microarray analysis, miRNAs that distinguish normal brain from medulloblastoma tissue were identified. As presented in the heat map, the dysregulated miRNAs are more closely associated with the medulloblastoma samples compared with the normal control samples. Additionally, by uploading the miRNAs from the first network to the IPA, it was identified that the dysregulated miRNAs were associated with key regulatory genes in tumorigenesis, including TP53, insulin-like growth factor 1 receptor, $\mathrm{AGO} 2$ and ERK1/2. The overexpressed miRNAs miR-17-5p and miR-182 (log fold change $>2$ ) have interactions with TP53; studies have demonstrated an association between these miRNAs and TP53 in tumorigenesis $(22,23)$. AGOs are associated with miRNAs, due to their function in the RNA induced silencing complex (RISC), wherein they use small RNA guides to recognize targets. AGO2 had an interaction with miRNAs that are dysregulated in medulloblastoma, including miR-17-5p, miR-128, miR-96, miR-433, miR-383 and miR-138.

The second network, including miR-217 and miR-216, which are upregulated in medulloblastoma, exhibits associations 
with key regulatory genes in medulloblastoma development, including TP53, SIRT1 and YBX1. SIRT1 is a well understood member of the sirtuin protein class, preventing cell aging and apoptosis of normal cells $(24,25)$. The effects of SIRT1 are controversial; the protein was considered to be a tumor promoter in neuroblastoma, prostate and skin cancer (26-28), whereas it was considered to be a tumor suppressor in breast and colon cancer $(29,30)$. Therefore, SIRT1 may serve a role in medulloblastoma and correlate with the formation and prognosis of human medulloblastomas (31). Recently, Dey et al (32) proved that YBX1 (YB1) is upregulated across human medulloblastoma subclasses and is required for medulloblastoma cell proliferation. Furthermore, the study identified that the SHH:YAP:YB1:IGF2 axis may be a powerful target for therapeutic intervention in medulloblastoma.

When the medulloblastoma samples were compared with the normal cerebellum, 9 dysregulated miRNAs were identified. The 9 miRNAs were uploaded to the IPA in order to perform the core analysis. The network involved 4 downregulated miRNAs and was associated with important regulators of tumorigenesis, including MET proto-oncogene (MET), AKT serine-threonine kinase 1 and sterol regulatory element-binding factor 1 (SREBF1). Previous evidence has demonstrated the association between aberrant MET signaling and medulloblastoma development $(33,34)$. Previous studies have revealed that overexpressed miR-206 may inhibit MET in lung cancer $(35,36)$, however the function of downregulated miR-206 in medulloblastoma is unknown. SREBF1 is a transcription factor, regulating lipogenesis, which is also involved in tumorigenesis (37). These network components alone are not sufficient to initiate medulloblastoma formation; the interactions between these dysregulated factors and additional genetic insults promote tumorigenesis.

In conclusion, based on the miRNA array data in the GEO database, miRNAs which are differentially expressed in medulloblastoma samples were identified. Certain dysregulated miRNAs have been confirmed; however, further research is required to verify the miRNAs which have not been previously identified. Additionally, the IPA core analysis, presenting the interaction of miRNAs and counterpart targets, provides a method to understand the tumorigenesis of medulloblastoma. The results of the present study may provide a potent target for therapeutic intervention or diagnosis in medulloblastomas.

\section{Acknowledgements}

The authors of the preset study would like to acknowledge the submitters of all these GEO data arrays and all the participants of this study. The present study was supported by the Lanzhou Science and Technology Bureau Project (grant nos. 2013-3-27 and 2015-3-86), Gansu Province Health Industry Research Project (grant nos. GSWSKY-2015-58 and GSWSKY-2015-58) and the doctoral research fund of Lanzhou University Second Hospital (grant no. ynbskyjj2015-1-02).

\section{References}

1. Louis DN, Ohgaki H, Wiestler OD, Cavenee WK, Burger PC, Jouvet A, Scheithauer BW and Kleihues P: The 2007 WHO classification of tumours of the central nervous system. Acta Neuropathol 114: 97-109, 2007.
2. Roussel MF and Hatten ME: Cerebellum development and medulloblastoma. Curr Top Dev Biol 94: 235-282, 2011.

3. Gottardo NG and Gajjar A: Current therapy for medulloblastoma. Curr Treat Options Neurol 8: 319-334, 2006.

4. Packer RJ, Rood BR and MacDonald TJ: Medulloblastoma: Present concepts of stratification into risk groups. Pediatr Neurosurg 39: 60-67, 2003.

5. Ferretti E, De Smaele E, Po A, Di Marcotullio L, Tosi E, Espinola MS, Di Rocco C, Riccardi R, Giangaspero F, Farcomeni A, et al: MicroRNA profiling in human medulloblastoma. Int J Cancer 124: 568-577, 2009.

6. Li KK, Pang JC, Lau KM, Zhou L, Mao Y, Wang Y, Poon WS and Ng HK: MiR-383 is downregulated in medulloblastoma and targets peroxiredoxin 3 (PRDX3). Brain pathol 23: 413-425, 2013.

7. Panwalkar P, Moiyadi A, Goel A, Shetty P, Goel N, Sridhar E and Shirsat N: MiR-206, a Cerebellum Enriched miRNA is downregulated in all medulloblastoma subgroups and its overexpression is necessary for growth inhibition of medulloblastoma cells. J Mol Neurosci 56: 673-680, 2015.

8. Weeraratne SD, Amani V, Teider N, Pierre-Francois J, Winter D, Kye MJ, Sengupta S, Archer T, Remke M, Bai AH, et al: Pleiotropic effects of miR-183 96 182 converge to regulate cell survival, proliferation and migration in medulloblastoma. Acta Neuropathol 123: 539-552, 2012.

9. Zhang Z, Li S and Cheng SY: The miR-183-96-182 cluster promotes tumorigenesis in a mouse model of medulloblastoma. J Biomed Res 27: 486-494, 2013.

10. Bartel DP: MicroRNAs: Genomics, biogenesis, mechanism, and function. Cell 116: 281-297, 2004.

11. Esquela-Kerscher A and Slack FJ: Oncomirs-microRNAs with a role in cancer. Nat Rev Cancer 6: 259-269, 2006.

12. Malumbres M: miRNAs versus oncogenes: The power of social networking. Mol Systems Biol 8: 569, 2012.

13. Adams BD, Kasinski AL and Slack FJ: Aberrant regulation and function of microRNAs in cancer. Curr Biol 24: R762-R776, 2014.

14. Cho YJ, Tsherniak A, Tamayo P, Santagata S, Ligon A, Greulich H, Berhoukim R, Amani V, Goumnerova L, Eberhart CG, et al: Integrative genomic analysis of medulloblastoma identifies a molecular subgroup that drives poor clinical outcome. J Clin Oncol 29: 1424-1430, 2011.

15. de Yébenes VG, Bartolomé-Izquierdo N, Nogales-Cadenas R, Pérez-Durán P, Mur SM, Martínez N, Di Lisio L, Robbiani DF, Pascual-Montano A, Cañamero M, et al: miR-217 is an oncogene that enhances the germinal center reaction. Blood 124: 229-239, 2014

16. Su J, Wang Q, Liu Y and Zhong M: miR-217 inhibits invasion of hepatocellular carcinoma cells through direct suppression of E2F3. Mol Cell Biochem 392: 289-296, 2014.

17. Li H, Zhao J, Zhang JW, Huang QY, Huang JZ, Chi LS, Tang HJ, Liu GQ, Zhu DJ and Ma WM: MicroRNA-217, down-regulated in clear cell renal cell carcinoma and associated with lower survival, suppresses cell proliferation and migration. Neoplasma 60: 511-515, 2013.

18. Xue Y, Abou Tayoun AN, Abo KM, Pipas JM, Gordon SR, Gardner TB, Barth RJ Jr, Suriawinata AA and Tsongalis GJ: MicroRNAs as diagnostic markers for pancreatic ductal adenocarcinoma and its precursor, pancreatic intraepithelial neoplasm. Cancer Genet 206: 217-221, 2013.

19. Deng M, Tang H, Zhou Y, Zhou M, Xiong W, Zheng Y, Ye Q, Zeng X, Liao Q, Guo X, et al: miR-216b suppresses tumor growth and invasion by targeting KRAS in nasopharyngeal carcinoma. J Cell Sci 124: 2997-3005, 2011.

20. Rachagani S, Macha MA, Menning MS, Dey P, Pai P, Smith LM, Mo YY and Batra SK: Changes in microRNA (miRNA) expression during pancreatic cancer development and progression in a genetically engineered KrasG12D; Pdx1-Cre mouse (KC) model. Oncotarget 6: 40295-40309, 2015.

21. Faraji F, Hu Y, Goldberger N, Wu G. Buetow KH, Zhang J and Hunter KW: Abstract A6: microRNA sequencing of AKXD recombinant inbred panel identifies miR-216b as a candidate metastasis suppressor in a murine model of breast cancer. Cancer Res 72: A6, 2012.

22. Kanaan Z, Rai SN, Eichenberger MR, Barnes C, Dworkin AM, Weller C, Cohen E, Roberts H, Keskey B, Petras RE, et al: Differential microRNA expression tracks neoplastic progression in inflammatory bowel disease-associated colorectal cancer. Hum Mutat 33: 551-560, 2012.

23. Lin LT, Chiou SH and Lee YJ: Abstract 3548: Study of the tumor suppressive machinery of arsenic trioxide-induced glioblastoma multiforme inhibition via microRNA-182-Sestrin2 regulatory circuit. Cancer Res 74: 3548, 2014. 
24. Kim D, Nguyen MD, Dobbin MM, Fischer A, Sananbenesi F, Rodgers JT, Delalle I, Baur JA, Sui G, Armour SM, et al: SIRT1 deacetylase protects against neurodegeneration in models for Alzheimer's disease and amyotrophic lateral sclerosis. EMBO 26: 3169-3179, 2007.

25. Law IK, Liu L, Xu A, Lam KS, Vanhoutte PM, Che CM, Leung PT and Wang Y: Identification and characterization of proteins interacting with SIRT1 and SIRT3: Implications in the anti-aging and metabolic effects of sirtuins. Proteomics 9: 2444-2456, 2009.

26. Chu F, Chou PM, Zheng X, Mirkin BL and Rebbaa A: Control of multidrug resistance gene mdrl and cancer resistance to chemotherapy by the longevity gene sirt1. Cancer Res 65: 10183-10187, 2005.

27. Hida Y, Kubo Y, Murao K and Arase S: Strong expression of a longevity-related protein, SIRT1, in Bowen's disease. Arch Dermatol Res 299: 103-106, 2007.

28. Yu W, Fu YC, Zhou XH, Chen CJ, Wang X, Lin RB and Wang W: Effects of resveratrol on $\mathrm{H}(2) \mathrm{O}(2)$-induced apoptosis and expression of SIRTs in H9c2 cells. J Cell Biochem 107: 741-747, 2009.

29. Firestein R, Blander G, Michan S, Oberdoerffer P, Ogino S, Campbell J, Bhimavarapu A, Luikenhuis S, de Cabo R, Fuchs C, et al: The SIRT1 deacetylase suppresses intestinal tumorigenesis and colon cancer growth. PLoS One 3: e2020, 2008 .

30. Wang RH, Zheng Y, Kim HS, Xu X, Cao L, Luhasen T, Lee MH, Xiao C, Vassilopoulos A, Chen W, et al: Interplay among BRCA1, SIRT1, and Survivin during BRCA1-associated tumorigenesis. Mol Cell 32: 11-20, 2008.

31. Ma JX, Li H, Chen XM, Yang XH, Wang Q, Wu ML, Kong QY, $\mathrm{Li}$ ZX and Liu J: Expression patterns and potential roles of SIRT1 in human medulloblastoma cells in vivo and in vitro. Neuropathology 33: 7-16, 2013.
32. Dey A, Robitaille M, Remke M, Maier C, Malhotra A, Gregorieff A, Wrana JL, Taylor MD, Angers S and Kenney AM: YB-1 is elevated in medulloblastoma and drives proliferation in Sonic hedgehog-dependent cerebellar granule neuron progenitor cells and medulloblastoma cells. Oncogene 35: 4256-4268, 2016.

33. Guessous F, Yang YZ, Johnson E, Marcinkiewicz L, Smith M, Zhang Y, Kofman A, Schiff D, Christensen J and Abounader R: Cooperation between c-met and focal adhesion kinase family members in medulloblastoma and implications for therapy. Mol Cancer Ther 11: 288-297, 2012.

34. Li Y, Lal B, Kwon S, Fan X, Saldanha U, Reznik TE, Kuchner EB, Eberhart C, Laterra J and Abounader R: The scatter factor/hepatocyte growth factor: c-met pathway in human embryonal central nervous system tumor malignancy. Cancer Res 65: 9355-9362, 2005.

35. Chen QY, Jiao DM, Wu YQ, Chen J, Wang J, Tang XL, Mou H, Hu HZ, Song J, Yan J, et al: MiR-206 inhibits HGF-induced epithelial-mesenchymal transition and angiogenesis in non-small cell lung cancer via c-Met /PI3k/Akt/mTOR pathway. Oncotarget 7: 18247-18261, 2016.

36. Chen QY, Jiao DM, Yan L, Wu YQ, Hu HZ, Song J, Yan J, Wu LJ, Xu LQ and Shi JG: Comprehensive gene and microRNA expression profiling reveals miR-206 inhibits MET in lung cancer metastasis. Mol Biosyst 11: 2290-2302, 2015.

37. Sun Y, He WW, Luo M, Zhou Y, Chang G, Ren W, Wu K, Li X, Shen J, Zhao X and Hu Y: SREBP1 regulates tumorigenesis and prognosis of pancreatic cancer through targeting lipid metabolism. Tumor Biol 36: 4133-4141, 2015.

38. Jinek M and Doudna JA: A three-dimensional view of the molecular machinery of RNA interference. Nature 457: 405-412, 2009. 